\title{
Energy-momentum tensor correlators and viscosity
}

\author{
Harvey Meyer* \\ Center for Theoretical Physics \\ Massachusetts Institute of Technology \\ Cambridge, MA 02139 U.S.A. \\ E-mail: meyerh@mit.edu
}

Collective flow has been observed in heavy ion collisions, with a large anisotropic component, and ideal hydrodynamic calculations had significant successful in describing the distribution of produced particles at the RHIC experiments. In order to account for this near ideal fluid behavior, the shear and bulk viscosity of the quark gluon plasma (QGP) must be computed from first principles in a regime where the QGP is not weakly coupled. In this talk I describe recent progress in computing energy-momentum tensor correlators on the lattice from which the viscosities can be extracted via Kubo formulas. I also show how to cumulate information from several channels, including at non-vanishing spatial momentum, in order to best constrain the viscosities. These methods should soon yield predictions at the higher temperatures that will be explored at the LHC experiments.

The XXVI International Symposium on Lattice Field Theory

July 14 - 19, 2008

Williamsburg, Virginia, USA

\footnotetext{
*Speaker.
} 


\section{Introduction}

Heavy ion collisions at RHIC have produced a rich phenomenology - see the contribution of R. Pisarski at this conference - which has revealed unexpected properties of the quark gluon plasma. Here I will be concerned with the thermodynamic and transport properties of the plasma in the range of temperatures $T_{c} \leq T \leq 4 T_{c}$. Hydrodynamics calculations [1, 2, 3] successfully described the distribution of produced particles in heavy ion collisions at RHIC [4, 5, 6, 7]. This early agreement between ideal hydrodynamics and experiment has been refined in recent times. On the theory side, the dissipative effects of shear viscosity $\eta$ have been included in full $3 \mathrm{~d}$ hydrodynamics calculations [8, 9, 10] and the sensitivity to initial conditions quantitatively estimated [11] for the first time. On the experimental side, the elliptic flow observable $v_{2}$, which is sensitive to the value of $\eta$ in units of entropy density $s$, is now corrected for non-medium-generated two-particle correlations [12]. The conclusion that $\eta / s$ must be much smaller than unity has so far withstood these refinements of heavy-ion phenomenology [11].

On the theory side, it is therefore important to compute the QCD shear viscosity from first principles to complete the picture. Furthermore, since the heavy ion collision program at LHC will probe the quark-gluon plasma at temperatures about a factor two higher, it is crucial to predict the shear viscosity at $\approx 3 T_{c}$, and from there to predict the size of elliptic flow, before experimental data is available.

A small shear viscosity is a signature of strong interactions, as I will explain in the next section; strong interactions in turn require non-perturbative computational techniques. In this talk I present the lattice calculation of the thermal correlators of the energy-momentum tensor in the Euclidean SU(3) pure gauge theory, and discuss methods to extract the shear and bulk viscosity from them. Computationally, the calculation is challenging enough without the inclusion of dynamical quarks, and physically, the thermodynamic properties of the QGP not too close to $T_{c}$ do not depend sensitively on the flavor content [13]. In perturbation theory [14], the ratio of shear viscosity to entropy density is $\mathrm{O}\left(\frac{1}{\alpha_{s}^{2}}\right.$ and there is only about $30 \%$ difference between the pure gauge theory and full QCD [15] at a fixed value of $\alpha_{s}(\eta / s$ is smaller in the pure gauge theory). It should be appreciated that this is not much for a quantity which is infinite in the Stefan-Boltzmann limit, and the difference is actually reduced when comparing the pure gauge theory and full QCD at a common value of $T / T_{c}$.

\section{The physical significance of viscosity}

Consider a dilute gas enclosed in a box, Fig. 1. A vertical velocity gradient $V / L$ can be established by pulling the upper plate horizontally, at the cost of overcoming a friction force per unit surface $F / S$. The macroscopic interpretation of that force is that neighboring layers of fluid are being dragged along. The microscopic mechanism is that molecules passing through a horizontal unit surface from above carry more longitudinal momentum than those coming from below. This generates a net transfer of momentum in the transverse direction. The longitudinal momentum of a molecule that passes through the unit surface is characteristic of the average longitudinal momentum in a slice located at a separation of order the mean free path $\ell_{\mathrm{mfp}}$ from the reference slice. The downward flux of molecules is clearly proportional to their density $n$. However the 


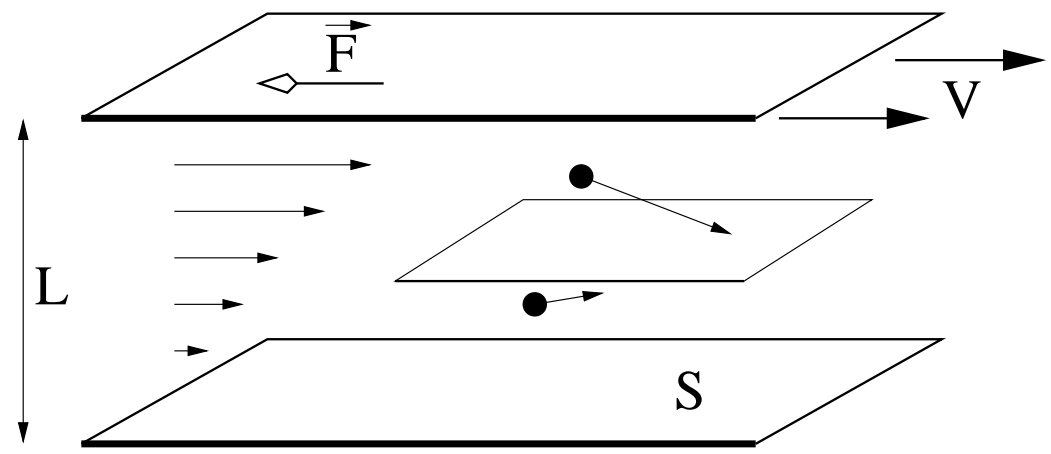

Figure 1: In the linear regime, the friction force per unit surface felt by a layer of fluid is proportional to the transverse velocity gradient. The proportionality coefficient is the shear viscosity: $F / S=\eta V / L$.

relation between the mean free path and the density, $\ell_{\mathrm{mfp}}=\frac{1}{n \sigma}$, cancels that dependence in the net momentum flux ( $\sigma$ is the interaction cross-section). The viscosity is then given by $\eta=\frac{\langle p\rangle}{3 \sigma}$, where $\langle p\rangle$ is the average momentum. The result is independent of the density $n$, at leading order in a density expansion.

A finite bulk viscosity has the effect of reducing the pressure of an expanding fluid, as compared to the adiabatic expansion, $P=P_{\text {equil. }}-\zeta \nabla \cdot v$ [16]. For a given equation of state, it can thus reduce the amount of radial flow in heavy ion collisions. It can also play an important role in cosmology [17]. In a dilute gas with two-body interactions, the bulk viscosity is proportional to the density of molecules, unlike the shear viscosity, and is therefore much smaller than the latter.

For further qualitative discussions of viscosity, see [18, 15].

\section{Methodology}

The set of operators that plays a central role in the calculation of the bulk and shear viscosities is the energy-momentum tensor. The Euclidean energy-momentum tensor for $\mathrm{SU}\left(N_{c}\right)$ gauge theories reads

$$
\begin{aligned}
T_{\mu v}(x) & =\theta_{\mu v}(x)+\frac{1}{4} \delta_{\mu v} \theta(x) \\
\theta_{\mu v}(x) & =\frac{1}{4} \delta_{\mu v} F_{\rho \sigma}^{a} F_{\rho \sigma}^{a}-F_{\mu \alpha}^{a} F_{v \alpha}^{a} \\
\theta(x) & =\frac{\beta(g)}{2 g} F_{\rho \sigma}^{a} F_{\rho \sigma}^{a} \\
\beta(g) & =-b_{0} g^{3}+\ldots, \quad b_{0}=\frac{11 N_{c}}{3(4 \pi)^{2}} .
\end{aligned}
$$

In contrast with Minkovsky space, $T_{0 i}=\theta_{0 i}$ is an antihermitian operator. In particular, $\left\langle T_{0 i}(x) T_{0 i}(0)\right\rangle<$ 0 for $x \neq 0$, and $P_{j}=i \int d^{3} \mathbf{x} T_{0 j}(x)$ is the usual momentum operator, for instance $P_{j}|\mathbf{q}\rangle=q_{j}|\mathbf{q}\rangle$ for a one-particle state.

The Euclidean correlators to be computed on the lattice are $\left(L_{0}=1 / T\right)$

$$
C_{\mu v, \rho \sigma}\left(x_{0}, \mathbf{q}, T\right)=L_{0}^{5} \int d^{3} \mathbf{x} e^{i \mathbf{q} \cdot \mathbf{x}}\left\langle T_{\mu v}(0) T_{\rho \sigma}\left(x_{0}, \mathbf{x}\right)\right\rangle .
$$

For diagonal correlators, the spectral functions are then defined by

$$
C_{\mu v, \mu v}\left(x_{0}, \mathbf{q}, T\right)=L_{0}^{5} \int_{0}^{\infty} \rho_{\mu v, \mu v}(\omega, \mathbf{q}, T) \frac{\cosh \omega\left(\frac{1}{2} L_{0}-x_{0}\right)}{\sinh \frac{\omega L_{0}}{2}} d \omega .
$$


(no summation over $\mu, v$ is implied here). The spectral functions are positive, $\rho(\omega, \mathbf{q}) / \omega \geq 0$, and odd, $\rho(-\omega, \mathbf{q})=-\rho(\omega, \mathbf{q})$. It is straightfoward to obtain a spectral representation for $\rho$, and thereby to show [19] that it is related to the Minkovsky-space retarded correlator via $\rho(\omega, \mathbf{q}, T)=$ $-\frac{1}{\pi} \operatorname{Im} G_{R}(\omega, \mathbf{q}, T)$. The Kubo formulas then relate the low-frequency behavior of $G_{R}(\omega, \mathbf{q}, T)$ with the shear and bulk viscosity, see [20] for a derivation. The shear and bulk viscosities are given by [21]

$$
\eta(T)=\pi \lim _{\omega \rightarrow 0} \frac{\rho_{12,12}(\omega, \mathbf{0}, T)}{\omega}, \quad \zeta(T)=\frac{\pi}{9} \sum_{k, \ell} \lim _{\omega \rightarrow 0} \frac{\rho_{k k, \ell \ell}(\omega, \mathbf{0}, T)}{\omega} .
$$

In Ref. [22], I defined the following moments of the spectral function $(n=0,1, \ldots)$ :

$$
\left\langle\omega^{2 n}\right\rangle \equiv L_{0}^{5} \int_{0}^{\infty} d \omega \frac{\omega^{2 n} \rho(\omega)}{\sinh \omega L_{0} / 2}=\left.\frac{d^{2 n} C}{d x_{0}^{2 n}}\right|_{x_{0}=L_{0} / 2}
$$

The latter equality implies that they are directly accessible to lattice calculations.

\section{Discretization of $T_{\mu \nu}$}

In the following sections I present results obtained with the 'clover' discretization of the energy-momentum tensor [23, 24]. I will use the anisotropic Wilson gauge actions, see for instance [25]. Indeed it has long been recognized that such a lattice presents advantages for the calculation of thermodynamics [25] and thermal correlation functions, in particular in charmonium calculations [26]. The downside is that more normalization factors are required.

The field strength tensor $\widehat{F}_{\mu v}(x)$ is given by

$$
\begin{aligned}
\widehat{F}_{0 k} & \left.=\frac{1}{8 a_{\sigma} a_{\tau}}\left(Q_{0 k}(x)-Q_{k 0}\right)\right) \\
\widehat{F}_{k l} & =\frac{1}{8 a_{\sigma}^{2}}\left(Q_{k l}(x)-Q_{l k}(x)\right) .
\end{aligned}
$$

It is the obvious generalization to the anistropic lattice of the definition found in [27]. We define the normalized operators as follows,

$$
\begin{aligned}
& a_{\sigma}^{3} a_{\tau} \theta_{00}(x)=\frac{1}{g_{0}^{2}} \operatorname{Re} \operatorname{Tr}\left\{\xi_{0} \widehat{Z}_{\mathrm{d} \tau}\left(g_{0}, \xi_{0}\right) \sum_{k}\left(a_{\sigma}^{2} a_{\tau}^{2} \widehat{F}_{0 k}(x)^{2}\right)-\xi_{0}^{-1} \widehat{Z}_{\mathrm{d} \sigma}\left(g_{0}, \xi_{0}\right) \sum_{k<l}\left(a_{\sigma}^{4} \widehat{F}_{k l}(x)^{2}\right)\right\}, \\
& a_{\sigma}^{3} a_{\tau} \theta(x)=-\frac{d g_{0}^{-2}}{d \log a_{\sigma}} \operatorname{Re} \operatorname{Tr}\left\{\xi_{0} \widehat{Z}_{\theta \tau}\left(g_{0}, \xi_{0}\right) \sum_{k}\left(a_{\sigma}^{2} a_{\tau}^{2} \widehat{F}_{0 k}(x)^{2}\right)+\xi_{0}^{-1} \widehat{Z}_{\theta \sigma}\left(g_{0}, \xi_{0}\right) \sum_{k<l}\left(a_{\sigma}^{4} \widehat{F}_{k l}(x)^{2}\right)\right\}, \\
& a_{\sigma}^{3} a_{\tau} \theta_{03}(x)=\frac{2 \widehat{Z}_{\mathbf{p}}}{g_{0}^{2}} \operatorname{Re} \operatorname{Tr}\left[\left(a_{\sigma}^{3} a_{\tau} \widehat{F}_{01} \widehat{F}_{31}\right)+\left(a_{\sigma}^{3} a_{\tau} \widehat{F}_{02} \widehat{F}_{32}\right)\right]
\end{aligned}
$$

Here $\xi_{0}$ is the bare anisotropy, and $\xi$ the renormalized one. In total, $\theta$ requires two normalization factors, and $\theta_{\mu \nu}$ requires five of them.

To fix the normalization factors of $\theta_{00}$ and $\theta$ we proceed as follows. Let $L$ be a physical length. Choose an ensemble ' 1 ' with $L_{0}=L_{1}=L_{2}=L_{3}=L$ and an ensemble ' 2 ' with $L_{0} / 2=L_{1}=L_{2}=$ $L_{3}=L$; then $F_{0} \equiv L^{4}\left(\left\langle\theta_{00}\right\rangle_{2}-\left\langle\theta_{00}\right\rangle_{1}\right)$ is an RGI quantity. The subtraction is necessary on the 
anisotropic lattice in order to cancel the mixing with the unit operator. Consider now an ensemble ' 3 ' where $L_{0}=L_{2}=L_{3}=L_{1} / 2=L$, and compute $F_{1} \equiv L^{4}\left(\left\langle\theta_{11}\right\rangle_{3}-\left\langle\theta_{11}\right\rangle_{1}\right)$. By symmetry between direction $\hat{1}$ and $\hat{0}, F_{0}=F_{1}$. This condition fixes the ratio of the two renormalization factors.

Similarly, requiring the equality of the RGI quantities $G_{0} \equiv L^{4}\left(\langle\theta\rangle_{2}-\langle\theta\rangle_{1}\right)$ and $G_{1} \equiv L^{4}\left(\langle\theta\rangle_{3}-\right.$ $\left.\langle\theta\rangle_{1}\right)$ fixes the relative size of the two normalization factors entering the trace anomaly operator $\theta$.

To fix the absolute size of the normalization factors, we equate the clover-discretized conformality measure $\langle\theta\rangle$ with the plaquette discretization, for which the normalization factors are known in terms of the lattice beta function [25] and the renormalization of the anisotropy [28]. For the case of $\theta_{00}$, we obtain its absolute normalization by calibrating its expectation value to the value of the entropy density obtained in [25] from the integral method.

The normalization factor of $T_{0 k}$ is determined by requiring that $C_{03,03}\left(x_{0}=1 / 2 T, \mathbf{0}, T\right)=s / T^{3}$. This equality can be checked explicitly in hydrodynamics and in free field theory.

\subsection{Cutoff effects in perturbation theory}

The cutoff effects affecting the Euclidean correlators can be studied, and, eventually, removed order by order in perturbation theory.

In the continuum and infinite volume limit, the two-point function of $\frac{1}{2}\left(T_{11}-T_{22}\right)$ equals the two-point function of $T_{12}$ for all $\mathbf{q}=(0,0, q)$. On the infinite lattice, discretizing either form yields a correlator that approaches the continuum limit with $\mathrm{O}\left(a^{2}\right)$ discretization errors. Figure 2 shows a comparison of the discretization errors affecting these two discretization schemes at $\xi=1$. Using the clover discretization of $T_{\mu \nu}$ at a given value of $N_{\tau}$, discretizing $T_{12}$ yields smaller discretization errors than discretizing $\frac{1}{2}\left(T_{11}-T_{22}\right)$, for all values of $x_{0}$. The figure also shows that the cutoff effects of the plaquette discretization used in [24] are almost identical to those obtained with the clover discretization of $T_{12}$.

In order to find the optimal range of anisotropies, we consider the cutoff effects on the tensor correlators at a fixed value of the spatial lattice spacing, $a_{\sigma} / L_{0}=$ fixed. We then vary the anisotropy between 1 and 4 . On the right panel of Fig. Z, we see that the sign of the cutoff effects changes for $1<\xi<2$, and goes to a finite value in the Hamiltonian limit, $\xi \rightarrow \infty$. It is clearly seen that any choice $\xi \geq 2$ reduces the cutoff effects significantly. It also appears that choosing $\xi>3$ does not reduce the cutoff effects further, because they are dominated by the coarseness of the spatial discretization. The cutoff effects are minimal near $\xi=2$, although, because of the sign change, this is partly accidental.

It should be noted that Eq. 3.6 holds in the continuum, and therefore the Euclidean correlator should in principle first be extrapolated in the standard way, before one attempts to reconstruct the spectral function. In practice, we often use data at finite lattice spacing instead, but one must then be careful not to use data at too small $x_{0} / a$, where cutoff effects become large. In the data presented in section 6 , we have reduced cutoff effects by dividing the non-perturbative lattice correlator by the treelevel lattice correlator and multiplying it by the continuum correlator.

\section{Analytic knowledge of the spectral function}

In this section we gather some of the known useful facts about the spectral functions. 

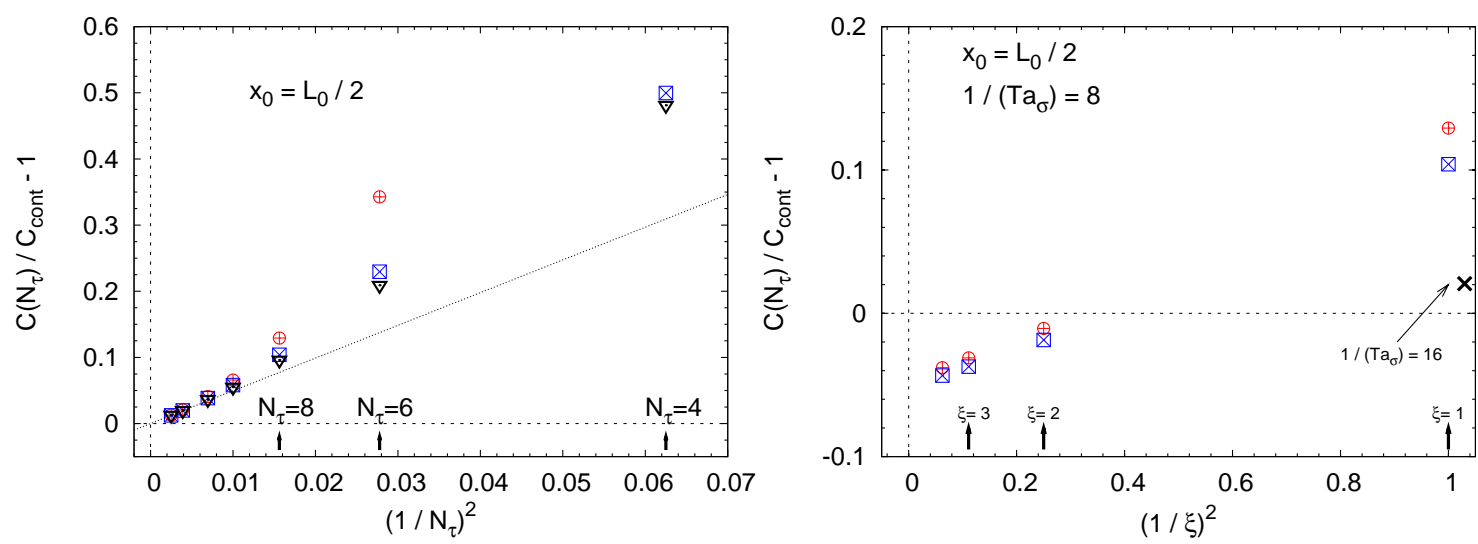

Figure 2: Left: The relative deviation of the $\mathbf{p}=0$ tensor correlator at finite lattice spacing from the continuum correlator. The spatial volume is infinite. The $\square$ 's are for the clover discretization of $T_{12}$ and the $\otimes$ for the clover discretization of $\frac{1}{2}\left(T_{11}-T_{22}\right)$. The $\nabla$ is the plaquette discretization used in [24]. Right: The cutoff effects on the tensor correlator for $x_{0}=L_{0} / 2$. The spatial lattice spacing $a_{\sigma}$ is held fixed, the temporal lattice spacing $a_{\tau}$ is varied between a quarter and one times $a_{\sigma}$. The $\square$ 's refer to $T_{12}$ and the $\otimes$ 's to $\frac{1}{2}\left(T_{11}-T_{22}\right)$. For comparison, the cutoff effect on an isotropic $N_{\tau}=16$ lattice is indicated by a cross.

\subsection{Ward identities}

The Ward identities that result from the conservation of the energy-momentum tensor, $\partial_{\mu} T_{\mu \nu}=$ 0 , imply, for $\mathbf{q}=q \hat{e}_{3}$,

$$
\begin{aligned}
\omega^{4} \rho_{00,00}(\omega, \mathbf{q})=-\omega^{2} q^{2} \rho_{03,03}(\omega, \mathbf{q}) & =q^{4} \rho_{33,33}(\omega, \mathbf{q}) \\
-\omega^{2} \rho_{01,01}(\omega, \mathbf{q}) & =q^{2} \rho_{13,13}(\omega, \mathbf{q}) .
\end{aligned}
$$

The first set of spectral functions is referred to as the 'sound channel' and the second as the 'shear channel' [20]. We therefore use the notation $\rho_{\text {snd }}(\omega, \mathbf{q}) \equiv \rho_{00,00}(\omega, \mathbf{q})$ and $\rho_{\text {sh }}(\omega, \mathbf{q}) \equiv \rho_{01,01}(\omega, \mathbf{q})$.

At zero temperature, Lorentz invariance imposes strong constraints on the analytic form of the spectral function. This is shown in some detail in the Appendix for $\rho_{\text {snd }}(\omega, \mathbf{q})$. In particular, the Euclidean correlator $C_{00,00}$ vanishes as $|\mathbf{q}|^{4}$ at $T=0$, whereas it generically only vanishes as $\mathbf{q}^{2}$ at finite temperature.

\subsection{Weak coupling predictions}

In [29], I computed the spectral functions in the free field theory approximation. They correspond to the result for photons, times the color factor $d_{A}=N_{c}^{2}-1$. At finite momentum, they are functions of two dimensionless parameters, $T x_{0}$ and $q / T$, and are linear combinations of polylogarithms. An example in the sound channel is shown on Fig. 3. Unlike in other channels illustrated in [29], the spectral function has a discontinuity at threshold, $\omega=q$. At zero-momentum, the spectral functions simplify considerably. For instance,

$$
\begin{aligned}
& \rho_{33,33}(\omega, \mathbf{0}, T)=\frac{2 d_{A}}{15(4 \pi)^{2}} \frac{\omega^{4}}{\tanh \frac{\omega}{4 T}}+\left(\frac{2 \pi}{15}\right)^{2} 3 d_{A} T^{4} \omega \delta(\omega) \\
& \rho_{12,12}(\omega, \mathbf{0}, T)=\frac{d_{A}}{10(4 \pi)^{2}} \frac{\omega^{4}}{\tanh \frac{\omega}{4 T}}+\left(\frac{2 \pi}{15}\right)^{2} d_{A} T^{4} \omega \delta(\omega)
\end{aligned}
$$


These free-field theory spectral functions of the fluxes contain an $\omega \delta(\omega)$ term, which expresses the conservation of a momentum flux, an unrealistic feature from the QCD point of view. To describe the dissipation of momentum flux, a more careful analysis is required, that was been carried out in [30, 16]. These studies show that the delta function becomes a peak of finite width, with a long tail in the $T_{k k}$ channel.

\subsection{Predictions of hydrodynamics}

If the momentum $\mathbf{q}=(0,0, q)$, the hydrodynamics predictions for the shear and sound channel are respectively [20]

$$
\begin{aligned}
\frac{\rho_{\mathrm{sh}}(\omega, \mathbf{q})}{\omega} \stackrel{\omega, q \rightarrow 0}{\sim} \frac{\eta}{\pi} \frac{q^{2}}{\omega^{2}+\left(\eta q^{2} /(\varepsilon+P)\right)^{2}}, \\
\frac{\rho_{\mathrm{snd}}(\omega, \mathbf{q})}{\omega} \stackrel{\omega, q \rightarrow 0}{\sim} \frac{\Gamma_{s}}{\pi} \frac{(\varepsilon+P) q^{4}}{\left(\omega^{2}-v_{s}^{2} q^{2}\right)^{2}+\left(\Gamma_{s} \omega q^{2}\right)^{2}},
\end{aligned}
$$

where

$$
\Gamma_{s}=\frac{\frac{4}{3} \eta+\zeta}{\varepsilon+P}
$$

is the sound attenuation length. The sound channel spectral function is illustrated on Fig. B.

\subsection{Strongly coupled $\mathscr{N}=4$ super-Yang-Mills}

The spectral functions of the $\mathscr{N}=4 S U\left(N_{c}\right)$ super-Yang-Mills theory were calculated by AdS/CFT methods in [31, 20] at infinite 't Hooft coupling and large number of colors. Those spectral functions are very smooth, and exhibit precisely the behavior generically predicted by hydrodynamics at low frequencies and momenta, as illustrated by Fig. 目. The sound peak in $\rho_{\text {snd }}$ is the dominant feature of the spectral function at least up to $q / 2 \pi T=0.6$. This is encouraging for lattice calculations, since the data presented in the next section already reach non-zero momenta as low as $q / 2 \pi T=0.125$.

\section{Numerical results}

We simulate the $\xi=2$ anisotropic Wilson gauge action with the two-level algorithm as described in [32 33]. We observe that correlators with a small, non-vanishing momentum are more accurate than at $\mathbf{q}=0$. The typical accuracy achieved on the correlators is $1 \%$, and in some cases it is as good as $0.5 \%$. Here we show results for the temperatures $1.27,1.52,2.52$ and 3.15 in units of $T_{c}$, respectively from lattices $12 \cdot 48^{3}, 10 \cdot 20^{3}, 10 \cdot 20^{3}$ and $8 \cdot 24^{3}$.

\subsection{Sound channel}

Due to the Ward identities, the correlators are all determined by the spectral function of the energy density. In particular, at fixed $x_{0}=1 / 2 T$,

$$
\begin{aligned}
C_{00,00}(q, T) & =\int_{0}^{\infty} d \omega \frac{\rho_{\mathrm{snd}}(\omega, q, T)}{\sinh \omega / 2 T}, \\
-C_{03,03}(q, T) & =\frac{1}{q^{2}} \int_{0}^{\infty} d \omega \omega^{2} \frac{\rho_{\mathrm{snd}}(\omega, q, T)}{\sinh \omega / 2 T},
\end{aligned}
$$



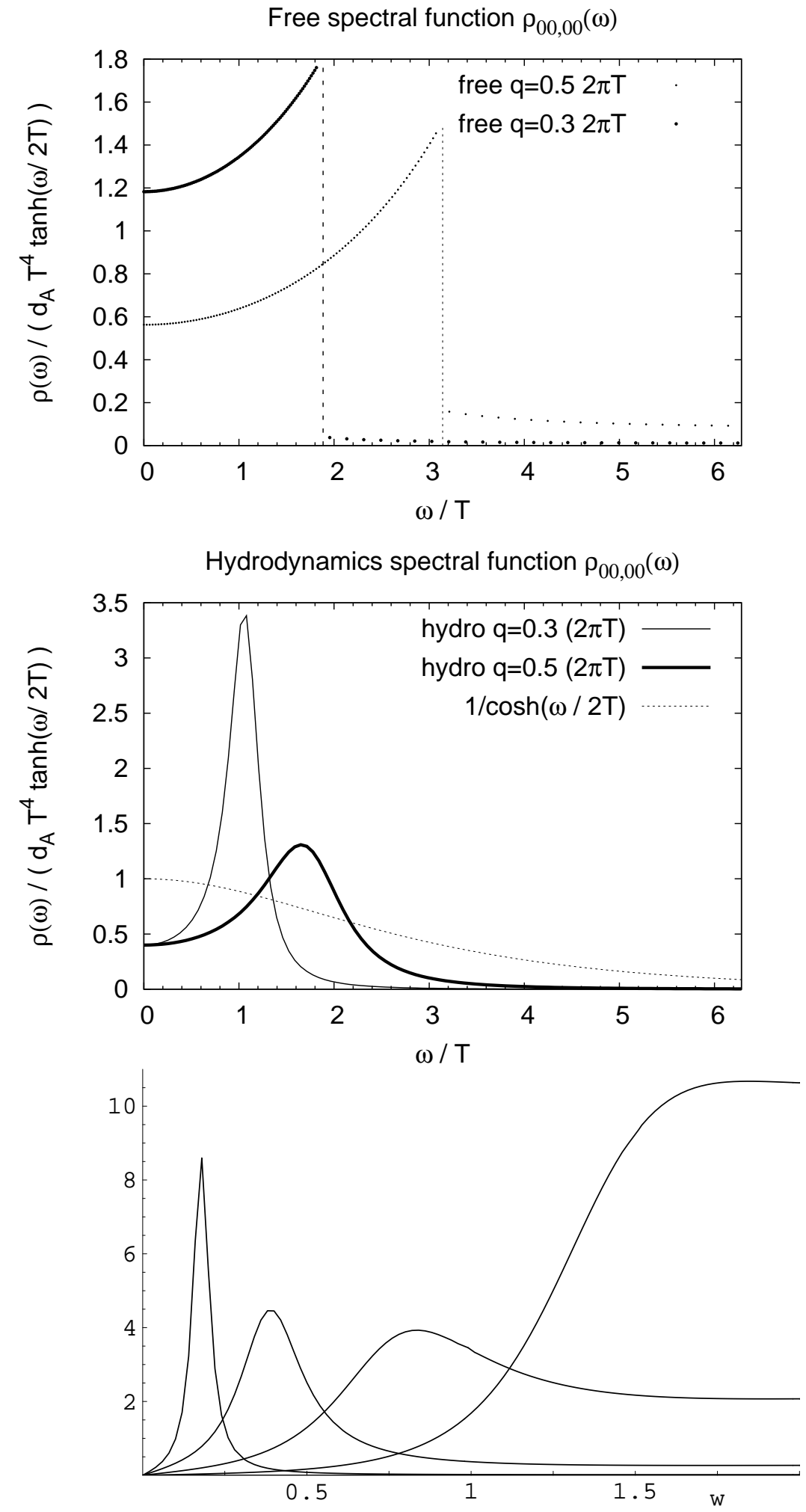

Figure 3: The $S U\left(N_{c}\right)$ gauge theory sound-channel spectral functions predicted by free theory (top). The functional form predicted by hydrodynamics (middle), for $v_{s}^{2}=\frac{1}{3}, s=\frac{3}{4} s_{S B}$ and $T \Gamma_{s}=\frac{1}{3 \pi}$. At the bottom, the exact result for $2 \rho /\left(\pi d_{A} T^{4}\right)$ in infinite-coupling, large- $N_{c}$ SYM theory, computed by AdS/CFT methods, is reproduced from [31]; (the $x$-axis variable is $w=\omega / 2 \pi T$ and the curves correspond to $q / 2 \pi T=0.3,0.6,1.0$ and 1.5). 

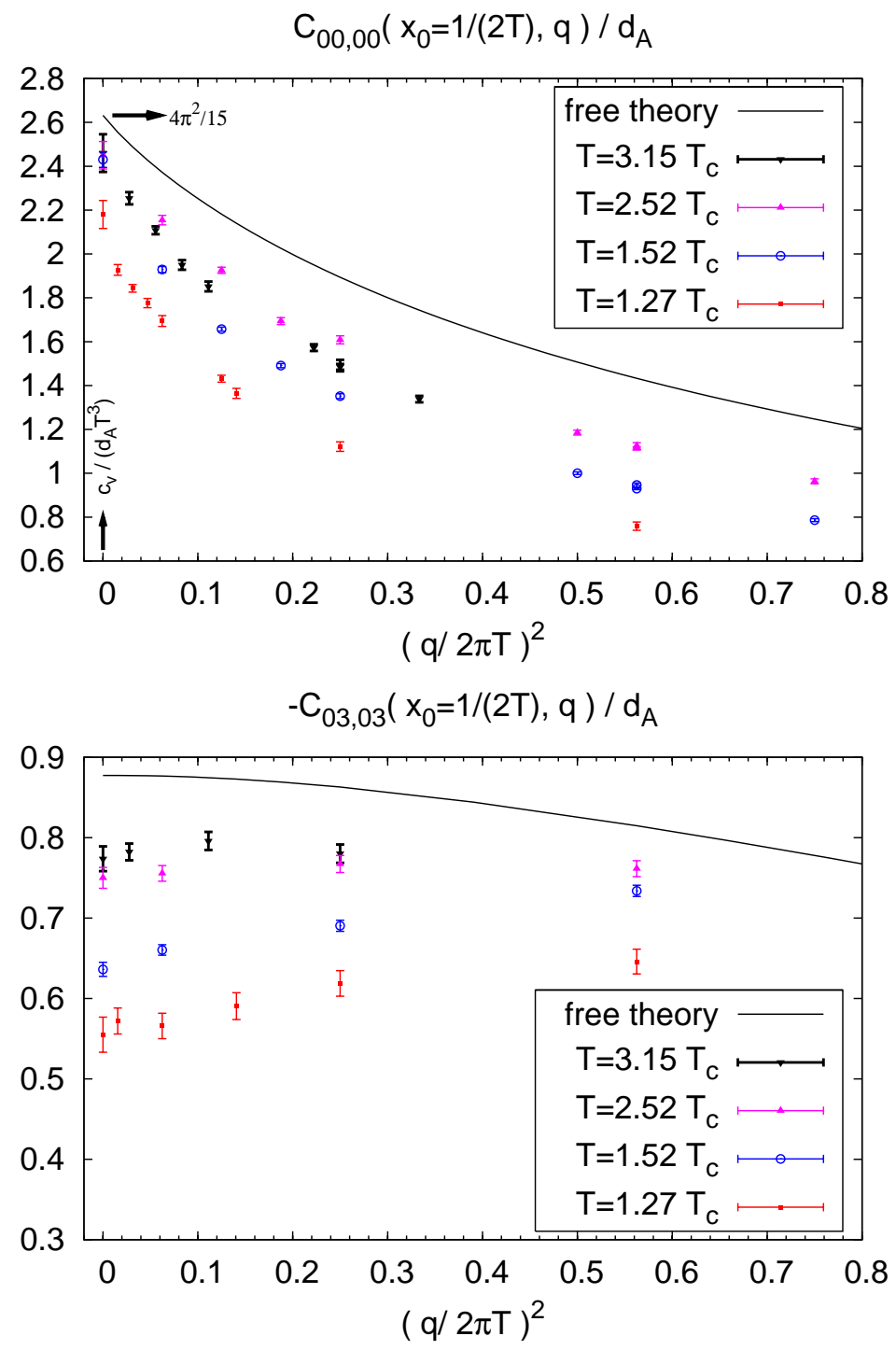

$\mathrm{C}_{33,33}\left(\mathrm{x}_{0}=1 /(2 \mathrm{~T}), \mathrm{q}\right) / \mathrm{d}_{\mathrm{A}}$

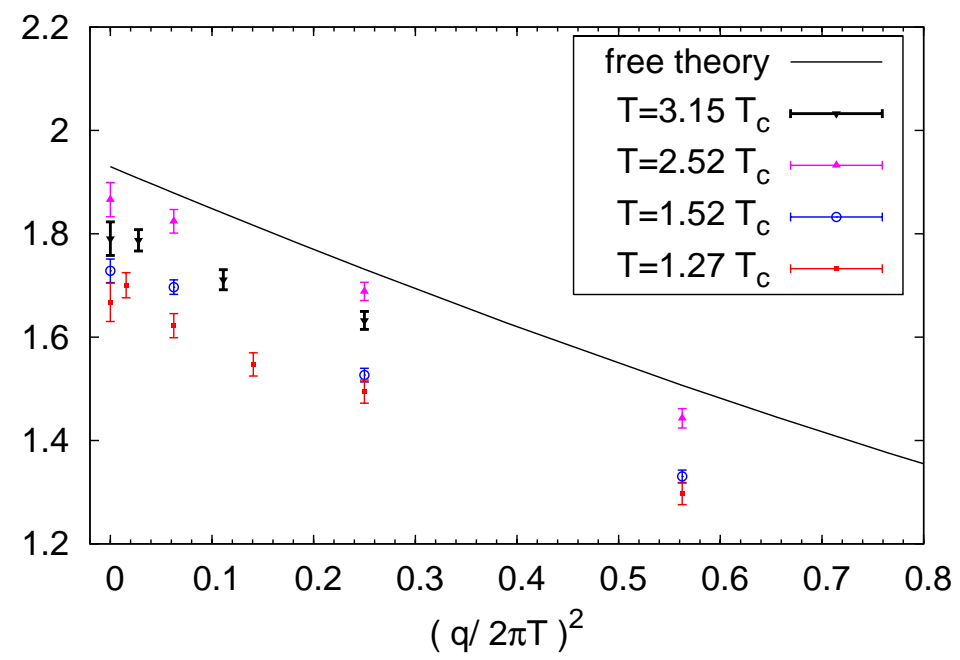

Figure 4: The sound-channel Euclidean correlators at $x_{0}=1 / 2 T$. 


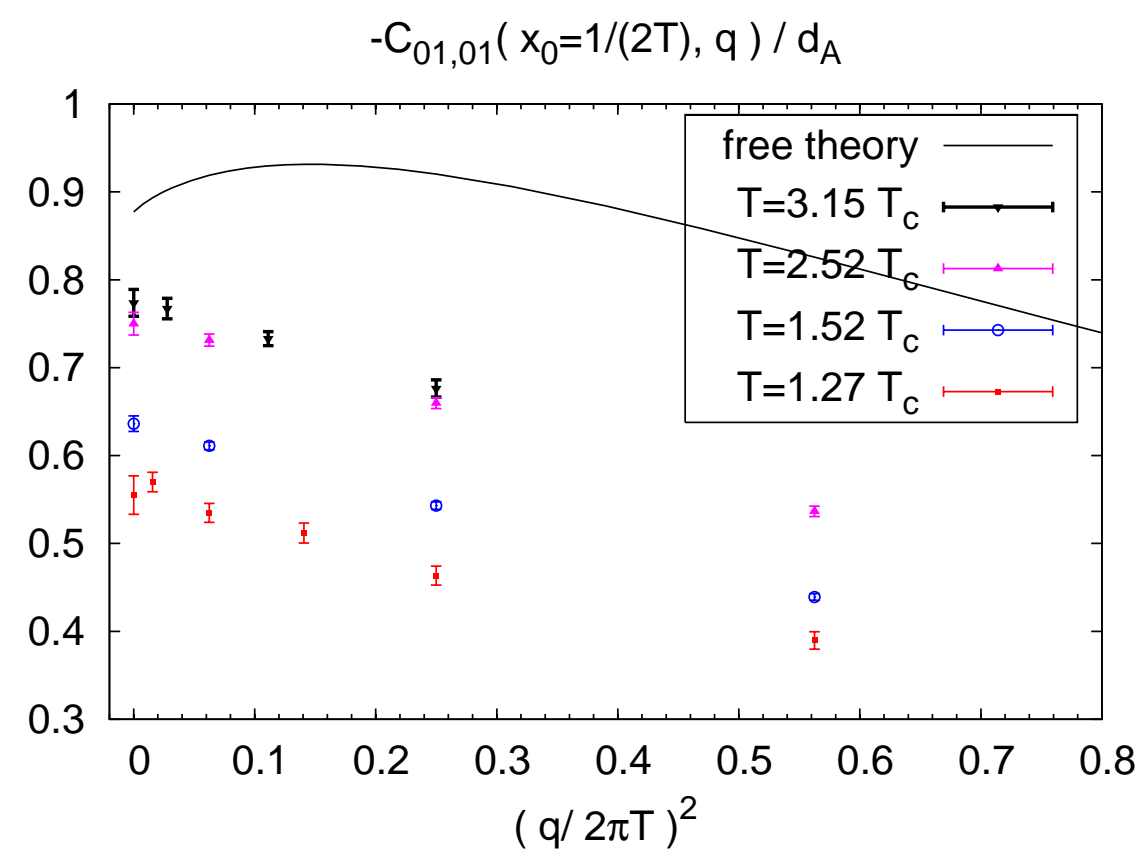

Figure 5: A shear-channel Euclidean correlator at $x_{0}=1 / 2 T$.

$$
C_{33,33}(q, T)=\frac{1}{q^{4}} \int_{0}^{\infty} d \omega \omega^{4} \frac{\rho_{\mathrm{snd}}(\omega, q, T)}{\sinh \omega / 2 T} .
$$

Computing $C_{03,03}$ and $C_{33,33}$ thus provides complementary information to $C_{00,00}$, without having to take finite differences in $x_{0}$, which necesarily leads to larger statistical and discretization errors.

Figure 4 displays the three correlators of the sound channel, as a function of spatial momentum $\mathbf{q}=q \hat{e}_{3}$. One remarkable feature is that below $2 T_{c},\left|C_{03,03}\right|$ increases with $q^{2}$ at small momentum. This is unlike the free theory prediction.

An Ansatz that interpolates between the hydrodynamic behavior at low frequencies and the perturbative behavior at high frequencies is

$$
\frac{\widehat{\rho}_{\mathrm{snd}}(\omega, q, T)}{\tanh \left(\frac{\omega}{2 T}\right)}=\frac{\frac{2}{\pi} \Gamma_{s}(\varepsilon+P) q^{4}}{\left(\omega^{2}-v_{s}^{2} q^{2}\right)^{2}+\left(\Gamma_{s} \omega q^{2}\right)^{2}}\left(1-\tanh ^{2} \omega / 2 T\right)+\frac{2 d_{A} q^{4}}{15(4 \pi)^{2}} \tanh ^{2} \omega / 2 T .
$$

Fitting to the correlator $C_{00,00}\left(\frac{1}{2 T}, \mathbf{q}, T\right)$ at $1.27 T_{c}$ for $q /\left(\frac{\pi T}{4}\right)=1, \sqrt{2}, \sqrt{3}$ and 2 , I obtain $T \Gamma_{s}=$ $0.15(10)$. This is compatible with my previous estimates [24, 22] for $\eta$ and $\zeta$.

In this (over-)simplified determination, I am relying on the fact that the integral of the sound peak multiplied by $1 / \cosh \frac{\omega}{2 T}$ is of the form $c_{v} T^{2}+\mathrm{O}\left(q^{2}\right)$, and that the $q^{2}$ term has some dependence on the width of the peak, itself given by the sound attenuation length. However, contributions from $\omega=\mathrm{O}(T)$ are not parametrically suppressed, although at these small momenta they are numerically very small both at weak coupling and in the strongly coupled SYM theory, see Fig. 3

A related point is that at order $q^{2}$, next-to-leading order hydrodynamic corrections to the sound peak enter as well, and those are not described in Ansatz (6.4). For instance, the sound velocity has a momentum dependence at next-to-leading order. At weak coupling [16] $\rho_{33,33}(\omega, \mathbf{0}, T) / \omega$ has a 


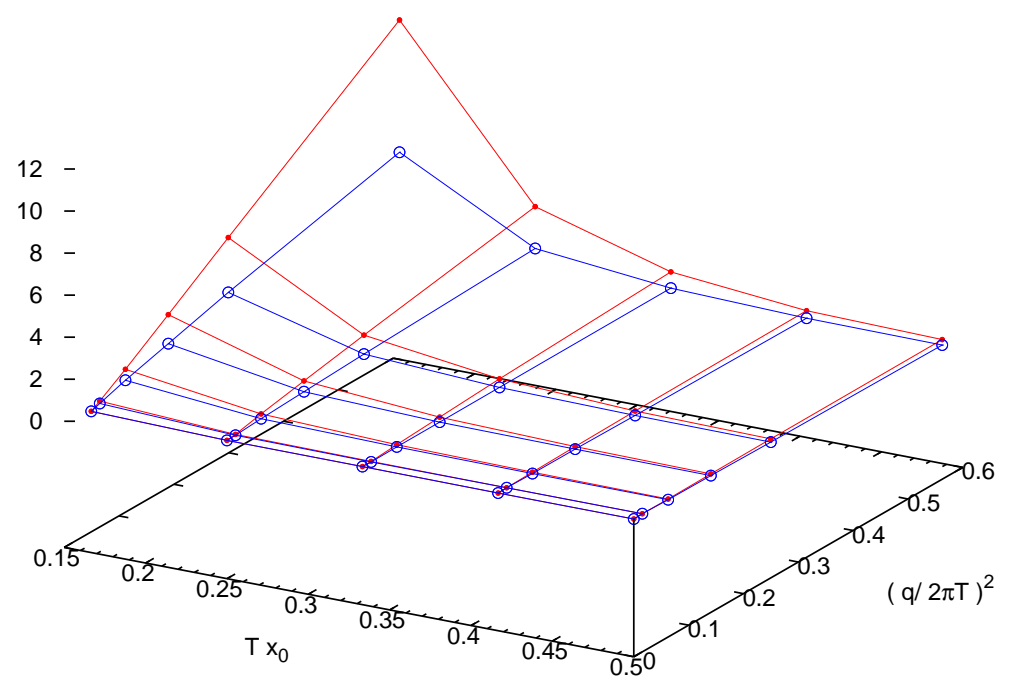

Figure 6: Momentum and Euclidean time dependence of the momentum density correlator $C_{01,01} / d_{A}$ and $C_{03,03} / d_{A}$ in the shear and sound channels, corresponding to open and closed circle respectively. Error bars are smaller than the symbols. The temperature is $1.27 T_{c}$.

peak whose width $\omega_{0}$ is related to the inverse of a relaxation time [34]. Since $\rho_{\text {snd }}=(q / \omega)^{4} \rho_{33,33}$, this effectively makes the sound peak narrower, typically like $\frac{1}{\left(\Gamma_{s} q^{2}\right)^{2}} \rightarrow \frac{1}{\left(\Gamma_{s} q^{2}\right)^{2}}+\frac{1}{\omega_{0}^{2}}$.

Further progress will come from simultaneously analyzing the $x_{0}$ and $q$-dependence of the correlator.

\section{Shear channel}

Similarly to the sound channel, at fixed $x_{0}=1 / 2 T$ and with spatial momentum $\mathbf{q}=q \hat{e}_{3}$,

$$
\begin{aligned}
-C_{02,02}(q, T) & =\int_{0}^{\infty} d \omega \frac{\rho_{\mathrm{sh}}(\omega, q, T)}{\sinh \omega / 2 T}, \\
C_{12,12}(q, T) & =\frac{1}{q^{2}} \int_{0}^{\infty} d \omega \omega^{2} \frac{\rho_{\mathrm{sh}}(\omega, q, T)}{\sinh \omega / 2 T} .
\end{aligned}
$$

Figure 5 displays the momentum correlator of the shear channel, as a function of spatial momentum. The derivative $\frac{d}{d q^{2}} C\left(\frac{1}{2 T}, q=0, T\right)$ is negative, unlike the free theory prediction.

\section{Discussion}

In the two-point functions of the momentum densities $T_{0 k}$, I found that below $2 T_{c}$ the first moment, $\frac{d}{d q^{2}} C_{0 k, 0 k}\left(\frac{1}{2 T}, q=0, T\right)$, is positive in the sound-channel $(k=3)$, and negative in the shear channel $k=1$. In free field theory, these signs are reversed. There is thus a qualitative difference between these non-perturbative correlators and the correlators in the weak coupling limit. By contrast, the correlation functions of the fluxes at vanishing spatial momentum are numerically not 
very different from the free-field prediction [24, 22, 19]. This is because they are dominated by the perturbative $\omega^{4}$ term in the spectral function at high frequency.

I pointed out the advantage of the conserved-charge correlators in terms of sensitivity to the low-frequency region $\omega \leq T$ region [29]. More generally, it is advantageous to constrain simultaneously the $\omega$ and $\mathbf{q}$ dependence of the spectral functions, since hydrodynamics makes a combined prediction in these two variables. Figure 6 displays the simultaneous $x_{0}$ and $q$ dependence of the momentum density correlators in the shear and sound channels. Thanks in part to treelevel improvement, the $\mathbf{q}=0$ correlator is $x_{0}$-independent to very good accuracy, as it should be. It is by fitting the whole surface parametrized by $\left(x_{0}, q\right)$ that one can best exploit the lattice data.

Recently, I have studied spatial correlation functions of the energy-momentum tensor [35]. The advantage of these observables is that no analytic continuation is required: they directly contain information on the space-like correlations present in the quark-gluon plasma. They can also be related to the spectral functions:

$$
C(\mathbf{r}, T)=\lim _{\varepsilon \rightarrow 0} \int \frac{d \mathbf{q}}{(2 \pi)^{3}} e^{i \mathbf{q} \cdot \mathbf{r}} \int_{0}^{\infty} d \omega e^{-\varepsilon \omega} \frac{\rho(\omega, \mathbf{q}, T)}{\tanh \omega / 2 T},
$$

which illustrates the wealth of information encoded in $\rho(\omega, \mathbf{q}, T)$. A hallmark of ordinary liquids is the short-range order observed (typically by neutron scattering) in the particle pair correlation function, which displays several gradually damped oscillations. One of the conclusions I reached is that the fluids with the smallest shear viscosity are those that are dense, but that are not ordered too strongly. The thermal contribution to the energy density correlations exhibits a non-monotonic behavior on distance scales of order $1 / T$ that is markedly stronger than in leading order perturbation theory. This may be the signature of a spatial ordering that is the quantum relativistic analogue of the short-range order characteristic of classical non-relativistic fluids. An attractive strategy to test the claimed liquid-like properties of the quark-gluon plasma [36] is to compare these spatial correlators with those computed in the strongly coupled SYM theory, which is known (from its spectral functions and small viscosity) to be an excellent fluid.

In conclusion, there has been much technical progress since the first calculation of the Euclidean correlators relevant to shear and bulk viscosity [21, 37, 24, 22]. The first accurate data in the shear channel allowed me to derive an upper bound on the shear viscosity, $\eta / s<1.0$ at $T<2 T_{c}$ under only weak assumptions [24]. To improve on this, the current emphasis is on exploiting simultaneously all the relevant lattice data, as well as the analytic properties of the spectral function known from perturbation theory in the ultraviolet and from hydrodynamics in the infrared. Spatial correlators provide complementary information that may help us understand the ability of the strongly coupled QGP to flow.

\section{Acknowledgments}

I thank Krishna Rajagopal and John Negele for valuable comments and encouragement. I thank Gert Aarts, Philippe de Forcrand, Berndt Müller and Derek Teaney for discussions. The $N_{\tau}=12$ simulations were performed on the BlueGene/L at MIT; I thank Andrew Pochinsky for his assistance in running it efficiently. The other simulations were performed on the desktop machines of the Laboratory for Nuclear Science, MIT. This work was supported in part by funds provided by the U.S. Department of Energy under cooperative research agreement DE-FG02-94ER40818. 


\section{A. The vacuum energy density correlator}

At zero temperature, we can exploit Lorentz invariance to constrain the analytic form of the spectral function. Following [38],

$$
\begin{aligned}
\int d^{4} x e^{i q x}\left\langle T_{\mu v}(x) T_{\rho \sigma}(0)\right\rangle & =\eta_{\mu v, \rho \sigma}(q) T(q \cdot q)+f_{\mu v, \alpha \beta}(q) T_{s}(q \cdot q) \\
\eta_{\mu v} & =q_{\mu} q_{v}-q^{2} \delta_{\mu v}, \quad f_{\mu v, \rho \sigma}=\eta_{\mu v} \eta_{\rho \sigma} \\
\eta_{\mu v, \rho \sigma} & =\eta_{\mu \rho} \eta_{v, \rho}+\eta_{\mu \sigma} \eta_{v \rho}-\frac{2}{3} \eta_{\mu v} \eta_{\rho \sigma} .
\end{aligned}
$$

This implies the following form of the energy density correlator in the mixed representation:

$$
C_{00,00}\left(x_{0}, \mathbf{q}\right)=|\mathbf{q}|^{4} \int_{-\infty}^{\infty} \frac{d q_{0}}{2 \pi} e^{-i q_{0} x_{0}}\left[\frac{4}{3} T\left(q_{0}^{2}+\mathbf{q}^{2}\right)+T_{S}\left(q_{0}^{2}+\mathbf{q}^{2}\right)\right] .
$$

In particular, the correlator satisfies

$$
\frac{\partial}{\partial x_{0}}\left(\frac{\partial}{\partial|\mathbf{q}|}-\frac{4}{|\mathbf{q}|}\right) C_{00,00}\left(x_{0}, \mathbf{q}\right)=|\mathbf{q}| x_{0} C_{00,00}\left(x_{0}, \mathbf{q}\right) .
$$

On the other hand, if

$$
v_{n}(\mathbf{q}) \equiv\left\langle n, \mathbf{q}\left|L^{-3 / 2} \int d^{3} \mathbf{x} e^{i \mathbf{q} \cdot \mathbf{x}} \widehat{T}_{00}(\mathbf{x})\right| \Omega\right\rangle
$$

the Källen-Lehmann representation of the correlator reads

$$
C_{00,00}\left(x_{0}, \mathbf{q}\right)=\sum_{n}\left|v_{n}\right|^{2}(\mathbf{q}) e^{-E_{n}(\mathbf{q}) x_{0}}
$$

On then easily shows that $\forall n$,

$$
E_{n}^{2}(\mathbf{q})=E_{n}^{2}(0)+\mathbf{q}^{2}, \quad\left|v_{n}\right|^{2}(\mathbf{q})=\frac{E_{n}^{2}(0) a_{n}^{2}|\mathbf{q}|^{4}}{2 E_{n}(\mathbf{q})}
$$

with $a_{n}$ a dimensionless number. In particular, all the $T=0$ contributions vanish as $\sim|\mathbf{q}|^{4}$ when $\mathbf{q} \rightarrow 0$. By the Ward identities of the energy-momentum tensor, $C_{33,33}\left(x_{0},|\mathbf{q}|\right)=|\mathbf{q}|^{-4} \frac{d^{4}}{d x_{0}^{4}} C_{00,00}\left(x_{0}, \mathbf{q}\right)$. Noticing that for a scalar state $S,\left\langle S\left|L^{-3 / 2} \int d^{3} \mathbf{x} \widehat{T}_{33}(x)\right| \Omega\right\rangle=\frac{1}{3}\left\langle S\left|L^{-3 / 2} \int d^{3} \mathbf{x} \widehat{\boldsymbol{\theta}}(x)\right| \Omega\right\rangle$, we then find the matrix element for the lightest glueball state,

$$
a_{0}=\frac{s}{3 M_{0}^{3}},
$$

where $s$, the matrix element of the trace anomaly, is defined and calculated in [39]. Similarly, realizing that $C_{33,33}\left(x_{0}, \mathbf{0}, T\right)=\frac{1}{9} C_{k k, l l}\left(x_{0}, \mathbf{0}, T\right)+\frac{4}{3} C_{12,12}\left(x_{0}, \mathbf{0}, T\right)$, we find that the matrix element $a_{2}$ corresponding to the tensor glueball is related to the matrix element $t$ defined in [39] by

$$
a_{2}=2 \sqrt{3} \frac{t}{3 M_{2}^{3}} .
$$




\section{References}

[1] P. F. Kolb, P. Huovinen, U. W. Heinz, and H. Heiselberg, Elliptic flow at SPS and RHIC: From kinetic transport to hydrodynamics, Phys. Lett. B500 (2001) 232-240, [hep-ph/0012137.

[2] P. Huovinen, P. F. Kolb, U. W. Heinz, P. V. Ruuskanen, and S. A. Voloshin, Radial and elliptic flow at RHIC: Further predictions, Phys. Lett. B503 (2001) 58-64, [hep-ph/0101136].

[3] D. Teaney, J. Lauret, and E. V. Shuryak, Flow at the SPS and RHIC as a quark gluon plasma signature, Phys. Rev. Lett. 86 (2001) 4783-4786, nucl-th/0011058.

[4] BRAHMS Collaboration, I. Arsene et. al., Quark gluon plasma and color glass condensate at RHIC? The perspective from the BRAHMS experiment, Nucl. Phys. A757 (2005) 1-27, [nucl-ex/0410020].

[5] B. B. Back et. al., The PHOBOS perspective on discoveries at RHIC, Nucl. Phys. A757 (2005) 28-101, [nucl-ex/0410022].

[6] STAR Collaboration, J. Adams et. al., Experimental and theoretical challenges in the search for the quark gluon plasma: The STAR collaboration's critical assessment of the evidence from RHIC collisions, Nucl. Phys. A757 (2005) 102-183, [hucl-ex/0501009].

[7] PHENIX Collaboration, K. Adcox et. al., Formation of dense partonic matter in relativistic nucleus nucleus collisions at RHIC: Experimental evaluation by the PHENIX collaboration, Nucl. Phys. A757 (2005) 184-283, [nucl-ex/0410003].

[8] P. Romatschke and U. Romatschke, Viscosity Information from Relativistic Nuclear Collisions: How Perfect is the Fluid Observed at RHIC?, Phys. Rev. Lett. 99 (2007) 172301, arXiv:0706.1522.

[9] K. Dusling and D. Teaney, Simulating elliptic flow with viscous hydrodynamics, Phys. Rev. C77 (2008) 034905, [arXiv:0710.5932].

[10] H. Song and U. W. Heinz, Suppression of elliptic flow in a minimally viscous quark- gluon plasma, Phys. Lett. B658 (2008) 279-283, arXiv: 0709.0742.

[11] M. Luzum and P. Romatschke, Conformal Relativistic Viscous Hydrodynamics: Applications to $R H I C$, arXiv:0804.4015.

[12] STAR Collaboration, B. I. Abelev et. al., Centrality dependence of charged hadron and strange hadron elliptic flow from sqrt(s) = $200 \mathrm{GeV}$ Au+Au collisions, Phys. Rev. C77 (2008) 054901, [arXiv:0801.3466].

[13] F. Karsch, Properties of the quark gluon plasma: A lattice perspective, Nucl. Phys. A783 (2007) 13-22, [hep-ph/0610024].

[14] P. Arnold, G. D. Moore, and L. G. Yaffe, Transport coefficients in high temperature gauge theories. II: Beyond leading log, JHEP 05 (2003) 051, [hep-ph/0302165.

[15] G. D. Moore, Transport coefficients in hot QCD, hep-ph/0408347.

[16] G. D. Moore and O. Saremi, Bulk viscosity and spectral functions in QCD, arXiv:0805.4201.

[17] S. Weinberg, Entropy generation and the survival of protogalaxies in an expanding universe, Astrophys. J. 168 (1971) 175.

[18] J. I. Kapusta, Viscous Properties of Strongly Interacting Matter at High Temperature, arXiv:0809.3746.

[19] H. B. Meyer, Computing the viscosity of the QGP on the lattice, arXiv:0805.4567. 
[20] D. Teaney, Finite temperature spectral densities of momentum and $R$ - charge correlators in $N=4$ Yang Mills theory, Phys. Rev. D74 (2006) 045025, [hep-ph/0602044].

[21] F. Karsch and H. W. Wyld, THERMAL GREEN'S FUNCTIONS AND TRANSPORT COEFFICIENTS ON THE LATTICE, Phys. Rev. D35 (1987) 2518.

[22] H. B. Meyer, A calculation of the bulk viscosity in SU(3) gluodynamics, Phys. Rev. Lett. 100 (2008) 162001, arXiv:0710.3717.

[23] M. Gockeler et. al., Lattice Operators for Moments of the Structure Functions and their Transformation under the Hypercubic Group, Phys. Rev. D54 (1996) 5705-5714, [hep-lat/9602029].

[24] H. B. Meyer, A calculation of the shear viscosity in SU(3) gluodynamics, Phys. Rev. D76 (2007) 101701, [arXiv:0704.1801].

[25] CP-PACS Collaboration, Y. Namekawa et. al., Thermodynamics of $S U(3)$ gauge theory on anisotropic lattices, Phys. Rev. D64 (2001) 074507, [hep-lat/0105012].

[26] M. Asakawa and T. Hatsuda, J/psi and eta/c in the deconfined plasma from lattice QCD, Phys. Rev. Lett. 92 (2004) 012001, [hep-lat/0308034.

[27] M. Luscher, S. Sint, R. Sommer, and P. Weisz, Chiral symmetry and $O(a)$ improvement in lattice QCD, Nucl. Phys. B478 (1996) 365-400, [hep-lat/9605038.

[28] T. R. Klassen, The anisotropic Wilson gauge action, Nucl. Phys. B533 (1998) 557-575, [hep-lat/9803010].

[29] H. B. Meyer, Energy-momentum tensor correlators and spectral functions, JHEP 08 (2008) 031, [arXiv:0806.3914].

[30] G. Aarts and J. M. Martinez Resco, Transport coefficients, spectral functions and the lattice, JHEP 04 (2002) 053, [hep-ph/0203177].

[31] P. Kovtun and A. Starinets, Thermal spectral functions of strongly coupled $N=4$ supersymmetric Yang-Mills theory, Phys. Rev. Lett. 96 (2006) 131601, hep-th/0602059.

[32] H. B. Meyer, Locality and statistical error reduction on correlation functions, JHEP 01 (2003) 048 , [hep-lat/0209145].

[33] H. B. Meyer, The Yang-Mills spectrum from a 2-level algorithm, JHEP 01 (2004) 030, [hep-lat/0312034.

[34] F. Karsch, D. Kharzeev, and K. Tuchin, Universal properties of bulk viscosity near the QCD phase transition, Phys. Lett. B663 (2008) 217-221, arXiv: 0711.0914 .

[35] H. B. Meyer, Density, short-range order and the quark-gluon plasma, arXiv:0808.1950.

[36] T. Hirano and M. Gyulassy, Perfect fluidity of the quark gluon plasma core as seen through its dissipative hadronic corona, Nucl. Phys. A769 (2006) 71-94, nucl-th/0506049].

[37] A. Nakamura and S. Sakai, Transport coefficients of gluon plasma, Phys. Rev. Lett. 94 (2005) 072305, [hep-lat/0406009.

[38] A. A. Pivovarov, Two-loop corrections to the correlator of tensor currents in gluodynamics, Phys. Atom. Nucl. 63 (2000) 1646-1649, [hep-ph/9905485].

[39] H. B. Meyer, Glueball matrix elements: a lattice calculation and applications, arXiv:0808.3151. 